Canadian

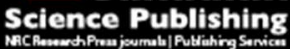

Canadian Geotechnical Journal Revue canadienne de géotechnique

\title{
HYDRAULIC BEHAVIOUR OF PARALLEL FIBRES UNDER LONGITUDINAL FLOW-A NUMERICAL TREATMENT
}

\begin{tabular}{|r|l|}
\hline Journal: & Canadian Geotechnical Journal \\
\hline Manuscript ID & cgj-2015-0213.R1 \\
\hline Danuscript Type: & Article \\
\hline Complete List of Authors: & $\begin{array}{l}\text { Nguyen, Thanh; University of Wollongong, Centre for Geomechanics and } \\
\text { Railway Engineering } \\
\text { Indraratna, Buddhima; University of Wollongong, }\end{array}$ \\
\hline Keyword: & $\begin{array}{l}\text { geoengineering, fluid-particle interaction, fibrous material, hydraulic } \\
\text { conductivity, permeability }\end{array}$ \\
\hline \multicolumn{2}{|c}{} \\
\hline
\end{tabular}




\title{
HYDRAULIC BEHAVIOUR OF PARALLEL FIBRES UNDER LONGITUDINAL FLOW-A NUMERICAL TREATMENT
}

\author{
Thanh Trung Nguyen \\ PhD student, \\ Centre for Geomechanics and Railway Engineering, University of Wollongong, \\ Wollongong City, NSW 2522, Australia
}

\section{Buddhima Indraratna}

BSc (Hons., Lond.), MSc (Lond.), DIC, PhD (Alberta), FTSE, FIEAust., FASCE, FGS

\begin{abstract}
Distinguished Professor of Civil Engineering, Faculty of Engineering and Information Sciences, Director, Centre for Geomechanics and Railway Engineering, University of Wollongong, Wollongong City, NSW 2522, Australia
\end{abstract}

Words: 8058

Submitted to: Canadian Geotechnical Journal

Corresponding author: Buddhima Indraratna (e-mail: indra@uow.edu.au) 


\title{
HYDRAULIC BEHAVIOUR OF PARALLEL FIBRES UNDER \\ LONGITUDINAL FLOW-A NUMERICAL TREATMENT
}

Thanh Trung Nguyen, Buddhima Indraratna

\begin{abstract}
Modelling fluid flow through fibrous porous materials has gained increasing attention from industry and research communities. The analytical and numerical methods are the two methods commonly adopted to predict the hydraulic characteristics of fibrous material during fluid flow, although to date, most techniques are conducted on the same assumption that the geometric features of fibres remain unchanged. In other words, the mutual interaction between fibre elements and fluid is ignored that undermines the actual working condition of fibres. This paper therefore presents a potential numerical approach which is capable of capturing the behaviour of fluid-solid system. Individual fibres are simulated by the Discrete Element Method (DEM) coupled with the concept of the Computational Fluid Dynamics (CFD), whereby the information contained in each phase is constantly exchanged and updated with each other. In comparison with conventional solutions, including the KozenyCarman (KC) fluid flow principle and other valid studies, the results show an acceptable agreement in predicting the hydraulic conductivity of a fibrous system. Subjected to laminar longitudinal flow, fibre motion is also evaluated with respect to varying bond stiffness and flow velocity. The study indicates a potential of the proposed technique in modelling drainage and filtration that are almost based on the hydraulic behaviour of fibrous porous geomaterials.
\end{abstract}

Keywords: geoengineering; fluid-particle interaction; fibrous material; hydraulic conductivity; permeability. 


\section{Introduction}

Fibrous porous materials have played an important role in a wide range of geoengineering applications, particularly in filtration, separation and drainage that are mostly related to the hydraulic conductivity of material. However, for many groups of fibres, especially for natural ones, the characteristics of hydraulic conductivity are not consistent due to their unstable porous structure, and that creates significant challenges in designs and applications. The long standing topic of seeking a comprehensive and flexible modelling technique for fibrous materials has received numerous concerns over the past decades. Most analytical approaches such as Gutowski et al. (1987); Gebart (1992); Yazdchi et al. (2011) estimate hydraulic conductivity by referring to the classical Kozeny-Carman concept which states that permeability depends on the porous characteristics, based on an assumption that a porous material is analogous to a system of capillaries channelled along the direction of flow and diameter. Note that although the Kozeny-Carman equation is applicable in a wide range of isotropic porous materials, it needs significant modification before it can be applied to unidirectional materials where transverse flow is significantly more constricted than longitudinal flow (Gebart 1992). For flow along a fibrous drain, a direct application of the KC equation is usually still acceptable (Sullivan 1942; Gebart 1992).

(1) $\quad K=\frac{\varepsilon d_{h}{ }^{2}}{16 k_{k}}$

In this equation, the coefficient $k_{k}$ is called the Kozeny constant that accounts for the geometric characteristics of the porous media; $\varepsilon$ is the porosity, and $d_{h}$ is the pore hydraulic diameter estimated from:

$$
d_{h}=\frac{4}{A_{o}} \frac{\varepsilon}{1-\varepsilon}
$$


In Equation (2), $A_{o}$ is the specific surface of the media and is defined as the ratio of the fluidsolid interfacial surface to the solid volume in a given fluid-solid system.

In this approach, the determination of Kozeny constant $k_{k}$ is the most controversial issue because it is mainly influenced by the geometric characteristics of porous media that are hard to define. Many previous studies have indicated a large range of Kozeny constant; for instance, Sullivan (1942) carried out a series of laboratory studies and summarised that $k_{k}$ of bundles of parallel fibres ranged from 0.806 for densely packed drill rods, with an $\varepsilon$ of 0.093 , to 10.57 for bundles of very loose wool fibres $(\varepsilon=0.98)$. Despite efforts to build a comprehensive and robust analytical solution to predict the permeability of fibrous materials, most current formulations are inherent in the Kozeny-Carman concept which requires the empirical constant $k_{k}$ to be determined. Ozgumus et al. (2014) carried out an impressive review surrounding the determination of Kozeny constant $k_{k}$, and they concluded that although $k_{k}$ can be suggested either as a constant or a function of porosity, the effect of porous characteristics on $k_{k}$ has not been well clarified.

Attributed to the rapid development of computer technology in recent decades, the numerical solution for fluid transport through fibrous porous media has gained more attention (Gebart 1992; Chen and Papathanasiou 2007; Tamayol and Bahrami 2008; Yazdchi et al. 2011). The flexibility of being able to describe geometric structures and remove the Kozeny constant in numerical approaches is obviously beneficial for practical designs and applications. Although various efforts to model the hydraulic conductivity of fibrous materials have been made over the past few decades, most current approaches assume the flow of fluid through a specific preform of porous media to be unchanged during simulation. The fibres in these models are actually treated as part of the boundary system which is normally assigned a constant set of parameters in the whole computation, but for many types of fibrous materials, especially for natural soft and light fibres, their fibrous structure is 
sensitive to external forces that cause variations in the pore characteristics, including interfibre distance, porosity, and effective flow path. Therefore, a framework which can simultaneously capture the behaviour of fluid and fibres is essential.

In recent times, the Discrete Element Method (DEM) has revealed its capacity to model the behaviour of material on the basis of analysing individual particles. Indeed, DEM has been used in a number of fields such as in applications to granular materials (Cundall and Strack 1979; Cheng et al. 2003; Bertrand et al. 2005) and rock mechanics (Potyondy and Cundall 2004; Cho et al. 2007). However, many studies (Zeghal and El Shamy 2004, 2008; O'Sullivan 2011) have shown that for a considerable number of geoengineering problems such as erosion, liquefaction which include two or more phases or interacting sub-systems, independently solving one phase or system is not sufficient and a consideration of the fluidparticle interaction is essentially required. This is why a great deal of effort has been put into describing particle-fluid interaction with major references to two approaches, namely the two-fluid model and coupling Computational Fluid Dynamics (CFD) with DEM (Zhou et al. 2010). In the CFD-DEM approach, DEM depicts the motion of discrete solid elements in parallel and interacting with the fluid flow simulated by CFD. Due to its major advantages, particularly in modelling fluid-solid systems, the coupled CFD-DEM has been recently applied to a variety of problems such as fluidisation, pneumatic conveying, pipeline flow, and gas cyclone, where a thorough review was carried out by Zhu et al., (2008). Although coupling CFD-DEM is still a state of the art simulation technique, it is a cost effective tool to numerically describe various mechanics and engineering problems.

In this paper, an application of CFD-DEM coupling on modelling the hydraulic behaviour of fibrous materials with respect to the fluid-fibre interaction is described. The fibres were considered to be distinct solid elements while the fluid flow was treated in conjunction with the continuity and conservation concepts of Navier-Stokes equation. A 
neutral framework called CFDEM was employed to exchange and update information between DEM and CFD platforms in every pre-defined number of time steps. Based on such a numerical scheme, a promising technique to simulate fluid-fibre system was then proposed.

\section{Theoretical background of coupled CFD-DEM approach}

The first application of the CFD-DEM coupling technique was carried out by Tsuji et al. (1993) to model the inviscid flow of gas through porous media while considering particle motion in two dimensions. In this work, particle motion was governed by Newton's law with respect to Discrete Element Method (Cundall and Strack 1979) in the micro-scale, while the Navier-Stokes equation was used to describe the flow of gas in a macro-scale. Encouraged by this interest, many researchers have improved the original CFD-DEM coupling technique and applied it to a number of fluid-particle problems as summarised by Zhu et al. (2008).

The keystone of this coupling technique is the inter-exchange of motion as well as interaction forces between two phases to ensure that the fluid and particles behave in conjunction with each other. The total fluid-particle interaction force $F_{f}$ represents all the forces of fluid acting on particles that might include the pressure gradient force, the viscous force, the virtual mass or added force, the Basset force and lift forces (Zhou et al. 2010). Selecting components of $F_{f}$ depends on the nature and specific conditions of each problem. The coupling process responsible for phase to phase communication is managed by a mutual framework called CFDEM that was developed by Goniva et al. (2010). Along the same time frame, while the particle motion is handled by a DEM framework named LIGGGHTS (Kloss and Goniva 2010), the fluid behaviour is governed by OpenFOAM (www.openfoam.com). LIGGGHTS is coupled with OpenFOAM on the basis of different theoretical backgrounds that are described below. 


\section{Governing equation of particles and fluid}

In the DEM proposed by Cundall and Strack (1979), the motion of a certain particle $i$ including translation and rotation, is governed by the following equations that are based on Newton's second law.

$$
\begin{aligned}
& m_{i} \frac{d U_{p, i}}{d t}=\sum_{j=1}^{n_{i}^{c}} F_{c, i j}+F_{f, i}+F_{g, i} \\
& I_{i} \frac{\omega_{p, i}}{d t}=\sum_{j=1}^{n_{i}^{c}} M_{c, i j}
\end{aligned}
$$

where $m_{i}$ and $I_{i}$ are the mass and inertia moment of particle $i$, respectively; $U_{p, i}$ and $\omega_{p, i}$ are the translational and angular velocities of particle $i ; F_{c, i j}$ and $M_{c, i j}$ are the contact force and torque exerting on particle $i$ by particle $j$ (or walls) while $n_{i}^{c}$ is referred to as the number of total contacts of particle $i$. The inter-particle contact behaviour is described by the either linear Hooke or non-linear Hertzian contact law which is adopted into DEM with respect to Coulomb's friction law and the concept of particle overlap during collisions (Cundall and Strack 1979). $F_{f, i}$ is the total fluid-particle interaction force acting on particle $i$ and $F_{g, i}$ is the gravitational force.

The governing equation employed in CFD is the locally averaged Navier-Stokes equation which describes the behaviour of pressure and velocity fields in the scale of a fluid cell. As described in past literature (Zhu et al. 2007; Zhou et al. 2010), there are two common distinct formulations of fluid flow equations, i.e., Model A and Model B. Model A assumes that the pressure is shared by the fluid and solid phases, while Model B only considers the fluid. Most investigations reported by Zhu et al. (2007) and Zhou et al. (2010) showed there was an insignificant difference between these two models, although Model A was more preferable, as noted by Zhou et al. (2010) and O'Sullivan (2011). In this study, Model A 
which incorporates the effects of particle volume fraction and distribution on pressure drop, was adopted.

$$
\begin{aligned}
& \frac{\partial \varepsilon}{\partial t}+\nabla \cdot\left(\varepsilon U_{f}\right)=0 \\
& \frac{\partial\left(\rho_{f} \varepsilon U_{f}\right)}{\partial t}+\nabla \cdot\left(\rho_{f} \varepsilon U_{f} U_{f}\right)=-\varepsilon \nabla p-f_{p}+\nabla \cdot(\varepsilon \tau)+\varepsilon \rho_{f} g
\end{aligned}
$$

where $U_{f}$ and $p$ are respectively, the velocity and pressure of fluid; $\tau$ and $\varepsilon$ denote the viscous stress tensor and porosity of computational fluid cell, respectively; $\rho_{f}$ is the fluid density and $g$ is the gravity constant; $f_{p}$ in Equation (6) is referred to as the mean volumetric particle-fluid interaction force exerted on the fluid cell by particles within the cell. The computation of $f_{p}$ is described by Equation (7) with respect to the weight factor $\varpi_{i o}$, which represents the volumetric portion of particle $i$ resided in cell $o$.

$$
f_{p, o}=\sum_{i=1}^{n_{p, o}} \varpi_{i o}\left(\frac{F_{p, i}}{V_{c, o}}\right)
$$

In the above, $F_{p, i}$ is the total force acting on particle $i ; V_{c, o}$ is the volume of fluid cell $o$ and $n_{p, o}$ is the total number of particles involved in cell $o$. The weight factor $\varpi_{i o}$ is defined as the ratio of the exact volumetric portion of particle $i$ in cell $o$ to the total volume of cell $o$. Clearly, $\varpi_{i o}$ is within $[0,1]$. If particle $i$ is completely positioned in cell $o$, then $\varpi_{i o}=1$.

The viscous stress tensor can be written in relation to the fluid viscosity $\mu_{f}$ and velocity $U_{f}$ as follows:

$$
\tau=\mu_{f}\left[\left(\nabla U_{f}\right)+\left(\nabla U_{f}\right)^{T}\right]
$$

The porosity (or void fraction) of a certain fluid cell is defined as the ratio of the void volume in cell to the total cell volume: $\varepsilon=V_{v} / V_{c}=1-V_{p} / V_{c}$ in which $V_{v}, V_{p}$ are the volumes of the void and particles occupied in cell, respectively; and $V_{c}$ is the volume of the cell. The 
porosity in a local cell can be computed on CFDEM based on several valid techniques such as the centre void fraction and divided void fraction methods (Zhao and Shan 2013). The divided void fraction method calculates the void fraction of a fluid cell by considering the actual portion of particle volume occupied in such a cell where particles overlap the boundary of the fluid cell. As a result it provides a more accurate value of the void fraction in the fluid cell, especially for a dilute and non-uniformly distributed particle system. Therefore, the divided void fraction technique was used in this study.

\section{Fluid-particle interaction forces}

In order to account for the effects of fluid on particle motion, the total fluid-particle interaction force $F_{f}$ which, in addition to the buoyancy force, can consist of the drag force, pressure gradient force, viscous force, and other unsteady forces (Zhu et al. 2007; Zhou et al. 2010), are introduced into the DEM framework. The drag force acting on particle $i$ positioned in fluid cell $o$, following De Felice's solution which was used and verified in studies such as Zhou et al. (2010), is calculated using Equation 9 as follows:

$$
F_{d, i}=\frac{1}{8} C_{d, i} \rho_{f} \pi D_{p, i}^{2} \varepsilon_{o}^{2}\left(\mathrm{U}_{f, o}-\mathrm{U}_{p, i}\right)\left|\mathrm{U}_{f, o}-\mathrm{U}_{p, i}\right| \varepsilon_{o}^{-\chi}
$$

where $U_{f, o}$ and $U_{p, i}$ represent, respectively, the averaged fluid velocity of cell $o$ and velocity of particle $i$ resided in cell $o$; $D_{p, i}$ is the diameter of the particle $i$ in which the drag force acts on. $C_{d, i}$ named as the fluid-particle drag coefficient, which is functional to Reynolds number of particle, is estimated by:

$$
C_{d, i}=\left(0.63+\frac{4.8}{\sqrt{R e_{\mathrm{p}, \mathrm{i}}}}\right)^{2}
$$

where the particle Reynolds number is determined as follows: 


$$
R e_{p, i}=\frac{\varepsilon_{o} \rho_{f} D_{p, i}\left|U_{f, o}-U_{p, i}\right|}{\mu_{f}}
$$

In Equation (9) for the drag force, the porosity function $\varepsilon^{-\chi}$ considers the presence of other particles in cell $o$ with the power factor $\chi$ functional to Reynolds number $R e_{p, i}$ of particle $i$, thus,

$$
\chi=3.7-0.65 \exp \left[-\frac{\left(1.5-\log _{10} R e_{p, i}\right)^{2}}{2}\right]
$$

The hydrostatic pressure gradient force accounts for the difference in pressure acting on a particle, and it might consist of the buoyancy force and components generated by the acceleration pressure gradient in the fluid. Zhou et al. (2010) combined these two terms of pressure gradient forces, while some may prefer to the separated force models used in the current CFDEM framework. The pressure gradient and buoyancy forces being exerted on particle $i$ with volume $V_{p, i}$ can be computed individually, as shown below.

$$
\begin{aligned}
& F_{\nabla p, i}=-(\nabla \cdot p) V_{p, i} \\
& F_{b, i}=-\rho_{f} g V_{p, i}
\end{aligned}
$$

The viscous force generated when a small solid object is moving inside a viscous environment is calculated as follows:

$$
F_{\nabla \cdot \tau, i}=-(\nabla . \tau) V_{p, i}
$$

Other forces such as the Basset and lift forces were omitted in this study because: $(i)$ the fluid was considered to be laminar and incompressible; (ii) although the motion of fibre particles can have both translational and rotational components, these fluid forces are insignificant compared with other forces such as the drag and pressure gradient forces considered in this study, particularly in laminar flow (Zhu et al. 2007; Zhou et al. 2010). 
Therefore, the total fluid-particle interaction force acting on particle $i$ was given by:

$$
F_{f, i}=F_{d, i}+F_{\nabla \cdot p, i}+F_{b, i}+F_{\nabla \cdot \tau, i}
$$

Note that the selection of fluid forces acting on particles represented in this study was applied for fibrous particles under laminar longitudinal flow and without interaction with soil particles. In many cases where the fibres are confined by surrounding soils and geostructures, components of the total fluid-particle interaction force can be different. For instance, viscous force can be ignored when a particle is confined and remains almost static during fluid flow, while the drag force which depends on the difference between $U_{f, o}$ and $U_{p, i}$ (Equation 9) is still considerable. The pressure gradient force is very important when there is a significant drop of pressure through a medium (i.e., low porosity). The drag and pressure gradient forces are usually the major contributors to the total fluid-particle interaction force (O'Sullivan 2011). On the other hand, when fibre particles have complex motions (e.g., significant rotation under turbulent flow), other fluid forces such as Basset and lift forces will need to be considered.

\section{Modelling the fibres}

Fibres are generally thread-like parts that can be classified as either natural or manmade. Regardless of their origin, their behaviour is generally considered in conjunction with continuum mechanics which treats material as continuous mass rather than discrete particles. Most fibres have a tensile strength that is much higher than bending and shearing, which explains why fibres can generally be spun (Mishra 2000). These characteristics make a fibrous structure sensitive to external forces.

Modelling a fibre by DEM is discretising a continuous string into a number of individual particles, such that bonding between these particles is applied with respect to the fibre's main characteristics, as mentioned above. In this study the so-called Parallel Bond 
Model (PBM) was used to simulate the fibres in DEM. The PBM has been used effectively to simulate a number of materials, including rock (Potyondy and Cundall 2004), cemented sand (Zeghal and El Shamy 2008) and geogrids (Ngo et al. 2014), based on elastic stress-strain relations. In addition to tensile, shear and bending behaviours of material that can be captured by the PBM, a review study conducted by Lisjak and Grasselli (2014) also indicated a wide application of the bond model in describing the fracturing process of brittle materials. This indicates a potential of the PBM in simulating a number of fibres, particularly natural fibres such as jute and bamboo, which have mainly linear stress-strain behaviour and brittle fracture (Defoirdt et al. 2010; Biswas et al. 2013). Fig. 1 illustrates how a stress-strain curve obtained from DEM using the PBM as summarized by Cho et al. (2007) can match well with the curve generalized from the experimental results obtained by Biswas et al. (2013) on jute and bamboo fibres.

As discussed by Potyondy and Cundall (2004), five parameters contribute to the properties of particles bonding, including the normal and shear stiffness per unit area $\left(k_{b n}\right.$ and $\left.k_{b s}\right)$; normal and shear strength $\left(\sigma_{b}\right.$ and $\left.\tau_{b}\right)$; and the bond radius multiplier $\lambda$ which defines a virtual shared area between two particles (Fig. 2). The value of $\lambda$ should be ranked from 0 to 1 as noted by O'Sullivan (2011). Generally, the radius of the bond region $R_{b}$ between two spherical particles $i$ with radius $R_{i}$ and $j$ with radius $R_{j}$ is estimated based on following condition:

$$
R_{b}=\lambda \min \left(R_{i}, R_{j}\right)
$$

The total force $F_{b, i j}$ and moment $M_{b, i j}$ withstood by the parallel bond can be discretised into normal (denoted by sub-script $n$ ) and shear (denoted by sub-script $s$ ) components, as follows: 


$$
F_{b, i j}=F_{b n, i j}+F_{b s, i j}
$$

$$
M_{b, i j}=M_{b n, i j}+M_{b s, i j}
$$

For the initial condition in which the relative displacement and rotation of two particles are zero, the bond force and moment are set to zero. Subjected to relative displacement- and rotation increments, $\Delta D_{n}, \Delta D_{s}, \Delta \theta_{n}, \Delta \theta_{s}$, the behaviour of particles bonding is governed by the linear elastic equations summarised below.

$$
\Delta F_{b n, i j}=-k_{b n} \Delta D_{n} A
$$

$$
\Delta F_{b s, i j}=-k_{b s} \Delta D_{s} A
$$

$$
\Delta M_{b n, i j}=k_{b s} J \Delta \theta_{n}
$$

where $A, I$ and $J$ are the area, moment of inertia, and polar moment of inertia of bond cross section, respectively. These parameters are given by:

$$
\Delta M_{b s, i j}=k_{b n} I \Delta \theta_{s}
$$

$I=\frac{1}{4} \pi R_{b}^{4}$

$$
\begin{aligned}
& A=\pi R_{b}^{2} \\
& I=\frac{1}{4} \pi R_{b}^{4} \\
& J=\frac{1}{2} \pi R_{b}^{4}
\end{aligned}
$$

With respect to beam theory, the maximum normal and shear stresses acting on the bond periphery are computed as follows:

$$
\sigma_{\text {max }}=\frac{-F_{b n, i j}}{A}+\frac{\left|M_{\mathrm{bs}, i j}\right|}{I} R_{b}
$$




$$
\tau_{\text {max }}=\frac{\left|F_{\mathrm{bs}, i j}\right|}{A}+\frac{\left|M_{\mathrm{bn}, i j}\right|}{J} R_{b}
$$

The bond is broken if either $\sigma_{\max }$ or $\tau_{\max }$ exceeds its corresponding strength, $\sigma_{b}$ and $\tau_{b}$. As a result the bond force and moment acting on the particle are removed.

In order to calibrate the parameters of the parallel bond for a certain material, primary tests such as tension, compression and shear tests are usually required. Because of various properties of fibres including soft fibres such as jute and flax, and hard structural fibres such as coir or others in a man-made group, the calibration process might require more than those tests. The axial compression and shear parameters of many types of fibres can be omitted, with only the tensile behaviour being considered, whereas the normal stiffness and strength are estimated by tensile testing, while the shear stiffness and strength are used as "dummy" parameters. Note that the determination of these parameters plays an important role in the behaviour of fibres under external forces.

Fig. 3 illustrates the behaviour of a single fibre simulated by DEM using the parallel bond with respect to different magnitudes of shear stiffness. In these investigations, the fibres had the same size $(0.005 \mathrm{~m}$ in diameter and $0.08 \mathrm{~m}$ in length), normal stiffness but different shear stiffness. One end of the fibre was fixed while the other end was free to move. Fig. 3a demonstrates the response of a hard fibre $\left(k_{b s}=1 \times 10^{9} \mathrm{~Pa} / \mathrm{m}\right.$ and density $\left.\rho_{p}=2500 \mathrm{~kg} / \mathrm{m}^{3}\right)$ subjected to an external force $F$. The fibre would bend until it reaches a steady state. The fibre having greater shear stiffness is less deformable due to bending. In Fig. 3b, behaviour of a soft and light fibre $\left(k_{b s}=1 \times 10^{2} \mathrm{~Pa} / \mathrm{m}\right.$ and density $\left.\rho_{p}=1000 \mathrm{~kg} / \mathrm{m}^{3}\right)$ suspending a mass (with the gravitational force $F_{g}$ equivalent to the external force $F$ applied on hard fibres) at one end is represented. Attributed to the low range of shear stiffness, soft fibres are generally less resistant to shear and bending forces. In fact, the motion shown in Fig. $3 b$ is similar to the motion of a simple pendulum system that consists of a string with a heavy mass suspended at 
one end. An ideal pendulum with a weightless, inextensible and perfectly flexible string $(0.08$ $\mathrm{m}$ in length) fixed at one end has a period of $0.567 \mathrm{~s}$, according to the well-known estimation $2 \pi \sqrt{l / g}$ where $l$ and $g$ are the length of string and gravity constant, respectively (Jaiswal and Singh 2013). This agreed well with the value (0.565 s) predicted by DEM using the PBM to capture the motion of the string. A slight deviation between the two values can be explained by several reasons, particularly: ( $i$ ) the string in DEM was not weightless and its mass contributed to the motion of the whole system; and (ii) the DEM string was not inextensible.

In addition, the elastic tensile stress-strain relationship governed by the parallel bond of DEM model in LIGGGHTS is seen in Fig. 4, which shows a good agreement between LIGGGHTS and PFC-3D used for geogrids (Ngo et al. 2014). Parameters of the PBM used to model geogrids are represented in Table 1.

A bundle of fibres can be generated by arranging individual fibres in a certain order. As mentioned in the previous part of this paper, various arrangements of fibres generating different porous characteristics of the drain system might influence the hydraulic transport of fibres. Within the scope of this study, the validity of CFD-DEM coupling on modelling the hydraulic behaviour of a fibre bundle with respect to fibre-fluid interaction was verified by the uniform triangular and rectangular distributions (Fig. 5) of fibres used for computational investigation. The properties of fibre material, including its size, density, and bond parameters were assumed to be uniform for the whole domain. These fibre characteristics were assumed with reference to previous studies (e.g., Mishra (2000)) on fibre engineering, particularly the fibre density ranging from 1000 to $2500 \mathrm{~kg} / \mathrm{m}^{3}$ and the equivalent diameters of fibre particles varying from $5 \times 10^{-4}$ to $8 \times 10^{-4} \mathrm{~m}$ that were used flexibly for different investigations in this study. 


\section{Correlation between DEM and real fibres}

Applying the DEM approach using spherical particles inevitably leads to several limitations in simulating fibre, including, $(i)$ the difficulty in achieving an extremely dense package of fibre, and (ii) the discrepancy between DEM fibre and real fibre shapes that results in a mismatch of hydraulic behaviour and fibre contact. The shape of a single fibre simulated in DEM is a string of discrete spheres that differ from their real shapes because most fibres have continuous and consistent shapes along their axes that enable them to pack densely (Chawla 2007). However, in the DEM approach using conventional contact models (i.e., Hooke and Hertzian models), minimum porosity which was achieved when the fibres were placed parallel and touched each other was 0.476 and 0.425 for rectangular and triangular arrangements, respectively. Note that these limitations of porosity were estimated based on several assumptions that include: $(i)$ treating the fluid cell in macro-scale and considering the fibre in micro-scale; (ii) the fibres consisted of a number of spheres; (iii) fibres were arranged in parallel and touched each other. Discussions regarding the porosity limitation of a fibre bundle can also be found in previous studies such as Tamayol and Bahrami (2009). Moreover, the discontinuity of DEM fibres along their axes caused conflict between the requirements of modelling the fibre contact and porosity. The diameter of spherical particles in DEM can be taken as exactly identical to the cross sectional size of real cylinder fibres to ensure the same contact behaviour in both systems, but it results in a mismatch in their porosity. Therefore this section will focus on solutions addressing these limitations.

\section{Fibre fraction of DEM fibrous system}

Due to the different pore structure in DEM and cylinder fibre systems, a quantified relationship between fibres in DEM and those in reality is imperative. Fig. 6 illustrates the 
diameter of real fibres converted to an equivalent diameter of DEM fibres, assuming that the cylinder fibre is a reasonable representation of real fibres. The equivalent diameter of particles in DEM can be estimated with respect to the consistency of porosity in both systems for which the following relationship was obtained.

$$
D_{f D E M}^{2}=\frac{3}{2} D_{f r}^{2}
$$

where $D_{f D E M}$ and $D_{f r}$ are the diameters of particles in DEM and cylindrical fibre in reality, respectively. The correlation shown in Equation (29) is to ensure that the porosity of fibres in the DEM simulation is the same as those obtained from real fibres. It is important to note that the porosity plays a key role in solving the Navier-Stokes equation by Finite Volume Method (FVM) that is currently applied to the CFD framework. Strictly speaking, the permeability of a fibre bundle also depends on the pore shape, which differs between the DEM and real fibre systems. Therefore, the impact of pore shape with respect to the $\mathrm{KC}$ concept may also be further considered. Particularly a fibre bundle simulated in DEM is actually a spherical arrangement with reference to a hydraulic diameter $d_{h}=4 / D_{f D E M}$, while a cylindrical fibre bundle corresponds to $d_{h}=6 / D_{f r}$. The theoretical application of the KC equation for spherical and cylindrical porous systems can be represented by Equations (30) and (31), respectively.

$$
\begin{aligned}
& K_{s}=\frac{D_{s}^{2} \varepsilon^{3}}{36 k_{k, s}(1-\varepsilon)^{2}} \\
& K_{c}=\frac{D_{c}^{2} \varepsilon^{3}}{16 k_{k, \mathrm{c}}(1-\varepsilon)^{2}}
\end{aligned}
$$

where the sub-scripts $s$ and $c$ refer to the spherical and cylindrical systems, respectively. For the same magnitude of porosity, the following relationship between the permeability of spherical and cylindrical porous mediums can be obtained. 


$$
\frac{K_{s}}{K_{c}}=\frac{2}{3} \frac{k_{k, c}}{k_{k, \mathrm{~s}}}
$$

Equation (32) explains the linkage between the permeability of spherical and cylindrical fibrous systems with respect to the $\mathrm{KC}$ approach. $K_{s}$ is the permeability obtained from the numerical model using spherical particles, and $K_{c}$ of cylindrical element system can then be estimated based on the value of $K_{s}$ according to Equation (32).

\section{Modified contact model for fibre simulation by DEM}

Given the differences between the characteristics of DEM and real fibres mentioned above, the concept of the conventional contact model must be modified to enable DEM fibres to attain a lower porosity and match the real fibre contact. Attributed to the overlap concept for soft particles proposed by Cundall and Strack (1979), the conventional contact model, including the linear Hooke and non-linear Hertzian models can be modified to achieve a higher fibre fraction. In DEM, the conventional contact model (Fig. 7a) will generate a positive interaction force between two particles whenever they overlap; and this prevents it from creating a lower porosity than the limited values mentioned above. The contact force $F_{i j}$ between the particles $i$ and $j$ is generally controlled according to the following spring-dashpot model (Bertrand et al. 2005).

$$
\begin{aligned}
& F_{c, i j}=F_{c n, i j}+F_{c s, i j} \\
& F_{c n, i j}=\mathrm{k}_{n} d_{i j}-\gamma_{c n} v_{c n, i j} \\
& F_{c s, i j}=\mathrm{k}_{s} s_{i j}-\gamma_{s} v_{s, i j}
\end{aligned}
$$

In the above, the subscripts $n$ and $s$ indicate the normal and shear components, respectively. $F, d, s, k, \gamma$ and $v$ are respectively the force, overlap, tangential displacement, stiffness, viscoelastic damping factor and relative velocity, with $i, j$ being referred to two particles in 
contact. The force components $F_{c n, i j}$ and $F_{c s, i j}$ are activated when the distance between particles $i$ and $j, d_{i j}<\left(R_{f D E M, I}+R_{f D E M, j}\right)$.

In order to let the DEM fibre bundle reach a higher value of fibre fraction and ensure the individual DEM fibres interact as closely as real fibres, the particles must overlap at a certain level without generating an interaction force. As Fig. 7a shows, the original contact model considers the contact forces in conjunction with the overlap of two DEM particles $i$ and $j$ having a radius of $R_{f D E M, i}$ and $R_{f D E M, j}$, respectively. However, the modified contact model in this study reduced the size of the DEM particles in contact with the real fibre diameter $D_{f r}$, while the diameter of DEM particles $\mathrm{D}_{\mathrm{fDEM}}$ was used to compute the porosity of the system. The contact force in the current model was hence generated when $d_{i j}<\left(R_{f r, i}+\right.$ $\left.R_{f r, j}\right)$ instead of $\left(R_{f D E M, i}+R_{f D E M, j}\right)$ in the conventional contact model, which enabled the DEM spherical fibres to be closer to each other, and thus achieve a higher fibre fraction. With respect to the modified contact model, the minimum porosity could reach significantly lower values than those (i.e., 0.476 and 0.425 for rectangular and triangular fibre arrangements, respectively) based on the conventional contact models.

\section{Simulation of fluid flow through fibrous material}

In this section, details of the coupled CFD-DEM technique used to model the hydrodynamic behaviour of fibrous material are presented. To verify this technique, the fibres with immobilisation of one ends were created. Significant motion of fibres might cause variation of fibre arrangement, and as a result the hydraulic conductivity of the system is unstable and incomparable to other studies which usually consider fibres to be static during fluid flow. Besides, in this study, the dimensionless permeability of a fibre bundle was investigated with respect to several assumptions, including $(i)$ the fibres were distributed uniformly; (ii) all the individual fibres in the bundle were homogeneous, and (iii) fluid flow 
was laminar. It is widely accepted that given an ordered array of fibres, the axial flow of fluid through a fibre bundle is independent of the size and shape of its cross-section (Endruweit et al. 2013). Therefore, to simplify, a cubic fluid domain with quadratic meshing was used with respect to the following desired targets: (i) porosity of the system; (ii) characteristics of fibre arrangement, and (iii) consistent CFD mesh and flow length. A typical fibre bundle modelled by DEM with reference to fluid flow can be seen in Fig. 8. The porosity of this system was subjected to change from 0.2 to 0.95 in this study, which corresponded to loose, medium, and dense fibre packing.

Fluid behaviour was managed by FVM based OpenFoam software which solves the governing equations of fluid in the control cells of CFD locally, therefore fluid domain discretisation plays a key role in the consistency and stability of numerical computations. With respect to the variation of porosity, the meshing fluid domain should be carried out while considering the porosity in each CFD cell and noting the correlation between the size of fluid cells and particles. Note that the current CFD-DEM coupling is based on the treatment that the fluid domain is evaluated in macroscopic variation while the particles are considered in microscopic variation. This means the dimensions of the CFD cell must be large compared to the size of the particles (Zeghal and El Shamy 2008). A wide range of studies used various size ratios of fluid cell to particle diameter, particularly from 1 to 5 (Chen et al. 2011; Smuts et al. 2012; Zhao and Shan 2013; Shan and Zhao 2014), whereas in this study a set of ratios between the CFD cell and particle diameter from 1 to 5 were also found to be suitable for numerical computation.

One of the most important factors contributing to the stability and accuracy of the numerical process is time discretisation, because a finer time frame guarantees a higher stability and accuracy of simulation but costs more in terms of computational time. The time step of a DEM framework was generally selected with reference to two critical values, $\Delta t_{h}$ 
and $\Delta t_{r}$ following the Hertzian and Rayleigh conditions (Li et al. 2005; Kloss et al. 2012) described by Equations (36) and (37), respectively.

$$
\begin{aligned}
& \Delta t_{h}=2.87\left(\frac{m_{e}^{2}}{R_{e} Y_{e}^{2} U_{\mathrm{p}, \max }}\right)^{0.2} \\
& \Delta t_{r}=\frac{\pi R}{(0.1631 \times v+0.8766)} \sqrt{\frac{\rho_{p}}{G}}
\end{aligned}
$$

In the above, $R$ is the average radius of particles; $v$ is Poisson's ratio; $\rho_{\mathrm{p}}$ denotes the density, and $G$ is the shear modulus of the particle. $U_{p, \max }$ is the maximum relative velocity of particles. $R_{e}, Y_{e}$ and $m_{\mathrm{e}}$ are the effective radius, Young's modulus, and mass of particles $i$ and $j$ in contact, respectively.

$$
\frac{1}{R_{e}}=\frac{1}{R_{i}}+\frac{1}{R_{j}}
$$

$$
\frac{1}{Y_{e}}=\frac{1-v_{i}^{2}}{Y_{i}}+\frac{1-v_{j}^{2}}{Y_{j}}
$$

$$
\frac{1}{m_{e}}=\frac{1}{m_{i}}+\frac{1}{m_{j}}
$$

While the time step of DEM framework $\Delta t_{p}$ was considered with respect to the critical time steps $\Delta t_{h}$ and $\Delta t_{r}$, the time step of CFD was determined in conjunction with the Courant number (OpenFOAM 2014) which is defined below.

$$
C o=\frac{\Delta t_{f}\left|U_{f}\right|}{\Delta x}
$$

where $\Delta t_{f}$ is the time step of numerical approximation in CFD; $U_{f}$ is the magnitude of fluid velocity through the cell, and $\Delta x$ is the size of the cell in the direction of fluid flow. The factor $C o$ should be less than unity for every cell of the fluid domain, which means the 
critical value of time step in the fluid solver can be written below as:

$$
\Delta t_{f}<\Delta t_{f_{-} c r i t}=\frac{\Delta x}{\left|U_{f}\right|}
$$

In CFD-DEM coupling, the time interval to exchange information between two phases is a crucial criterion to ensure the stability and accuracy of the simulation. The closer exchange and update between continuum (CFD) and discrete (DEM) platforms results in higher accuracy, but it is more costly in terms of computational time, whereas information on the solid phase such as motion and velocity might be lost or updated incorrectly in the CFD framework if the time interval is not small enough. The size of the time interval depends mainly on the nature of the problem simulated and might range over a wide scale, for example from 10 (Goniva et al. 2010) to 1000 (Zhao and Shan 2013). In this study, considering the properties of fibres and the CFD meshing details mentioned above, time steps of $10^{-7} \mathrm{~s}$ and $10^{-5} \mathrm{~s}$ were used for the DEM and CFD frameworks, respectively. The fluidparticle interaction was routine in every 100 DEM time steps $\Delta t_{p}$.

Fluid flow was set from one side of the flow path to the other, through fibrous media. The flow velocity was as small as possible to ensure proper laminar flow behaviour (Trussell and Chang 1999), and one ends of the fibres were fixed. To ensure the fully developed condition of flow, the fibre bundle should be long enough, i.e., $L / D_{f}>40$ (Tamayol and Bahrami 2010) where $L$ is the length of fibre bundle. In addition, extended flow paths before and after fibre domain were included (Fig. 8b), and the walls of CFD domain were assigned as slip boundaries to guarantee no friction of flow and uniform distribution of velocity. The drop in pressure could be extracted from the results of numerical computation, and Darcy's law which describes the permeability of porous media relative to the flow velocity and drop in pressure was used to calculate the longitudinal permeability of fibrous material. 


$$
U_{f, s}=-\frac{K}{\mu_{f}} \nabla p
$$

where $U_{f, s}$ and $\mu_{f}$ are the superficial velocity and the dynamic viscosity of fluid, respectively; $\nabla p$ is the pressure gradient accounting for the pressure drop $\Delta p$ over the length of flow path; and $K$ is the permeability. The dimensionless permeability $K / D_{f r}^{2}$ can then be calculated based on the $U_{f, s}$ and $\nabla p$ obtained from the numerical simulation.

\section{Results and Discussion}

\section{Hydraulic conductivity}

To verify the current numerical technique with conventional methods which consider fibres to be static during fluid flow, the DEM fibres with high bond stiffness were used (i.e., $k_{b n}=1 \times 10^{11} \mathrm{~Pa} / \mathrm{m}$ and $\left.k_{b s}=1 \times 10^{9} \mathrm{~Pa} / \mathrm{m}\right)$. Fig. 9 shows the variation of fluid fields, including the velocity and pressure, when flowing along fibre bundle with a porosity of 0.47 . In this series of studies, a superficial velocity $U_{f, s}=0.001 \mathrm{~m} / \mathrm{s}$ was used to ensure fluid flow in the Darcy regime. The relationship between superficial and duct velocities $\left(U_{f, s}\right.$ and $U_{f, d}$, respectively) is described as $U_{f, s}=\varepsilon U_{f, d}$, and shown in Fig. 9.

The CFD framework based on FVM that was used to solve the averaged NavierStokes equation locally in the individual fluid cell was able to describe the distribution of fluid velocity for varying arrangements of fibres, as discussed by Tamayol and Bahrami (2008) using static fibres. Fig. 10 represents the velocity distributions of fluid flow through the triangular and square arrangements of fibres modelled by the CFD-DEM coupling technique, and indicates its ability to capture the variation of fluid velocity due to the fibres being re-arranged. The motion of fibrous particles due to external force causes variations of porosity in the fluid cell, and as a result, the fluid fields changes by solving the Navier-Stokes equation. A finer mesh of CFD means a larger number of fluid cells generated and that can 
provide a more accurate description of fluid velocity distribution, but note that the current technique considers the CFD cell in a macro scale unlike the micro dimension of the DEM particles. This approach (coarse-grid approximation method, Tsuji et al. (1993)) cannot describe the flow within each individual fluid cell of the porous system (O'Sullivan 2011), as a result, limits the simulation to capture velocity distribution effectively, particularly for the densely packed fibres where the porosity is more homogeneous. It is evident that a uniform porosity in all fluid cells gives tangible outcomes after the locally averaged Navier-Stokes equation is solved, regardless of how the fibres are arranged. Therefore, in this study the velocity of the fluid in a dense fibre bundle could be considered uniform in all the fluid cells.

Fig. 11 represents the dimensionless longitudinal permeability of the squarely arranged fibres obtained from the CFD-DEM coupling technique; it shows a good agreement with those gained from Kozeny-Carmen solution and other studies, including the experimental works by Sullivan (1942); the numerical simulation by Tamayol and Bahrami (2008); and the analytical solutions by Happel (1959) and by Tamayol and Bahrami (2009). Note that in this investigation the fibres had insignificant motion and pressure was shared by the solid and fluid phases in the system, while other conventional studies used completely static fibres. The results show a good match between the coupled CFD-DEM technique and other analytical and numerical simulations for medium and loose fibre fraction $(0.4<\varepsilon<$ 0.95), but there is a relative deviation when the porosity is less than 0.4 . All the analytical and numerical methods presented agree with the experimental work of Sullivan (1942) within the porosity of very loose fibre bundle $(\varepsilon>0.9)$ and only begin to deviate from this experimental trend when $\varepsilon<0.9$. The Hapel (1959) and KC analytical methods, which do not consider the velocity distribution of fluid flow, share the same trend of permeability, while other studies (Tamayol and Bahrami 2008, 2009) report higher values of hydraulic conductivity when the porosity is less than 0.4 . For densely packed fibres where the current CFD-DEM coupling 
approach is not effective to capture the velocity distribution, the permeability curve by CFDEM is as close as $\mathrm{KC}$ and Hapel (1959) which assumes a uniform velocity distribution of fluid flow.

An investigation into the hydraulic conductivity of a fibrous medium in relation to the fibre arrangements including the rectangular and triangular distributions of fibres was conducted by CFD-DEM coupling. Given a parallel fibrous bundle with a uniform porosity, the results indicated that the longitudinal permeability of such a system slightly depended on the template of fibre distribution. Fig. 12 which represents a typical result obtained from the CFD-DEM coupling technique for rectangular fibre distribution, shows an insignificant variation of the hydraulic conductivity against the arrangement ratio $S_{y} / S_{x}$ of a fibrous system with $S_{x}$ and $S_{y}$ are the fibre distances in $x$ and $y$ directions (Fig. 5). This is reasonable because for unidirectional flow with a slippery velocity boundary condition and a uniform distribution of fluid velocity, flow parallel to the fibres can be treated as flow through a group of capillaries that is mainly related to the total cross section area of pores (Carman 1937; Tamayol and Bahrami 2009).

\section{Fluid-particle interaction forces}

Particles forming fibres are subjected to a number of forces which determine the motion of the whole fibre during fluid flow (Fig. 13a). For upward laminar fluid flow along the fibres in this study, the static buoyancy force and other fluid interaction forces acting on a fibre particle were in the same direction, and given the parallel arrangement of fibres, the contact force of fibre particles could be negligible. A study on the relationship between the total fluid-particle interaction force and flow velocity was carried out by CFD-DEM coupling with the uniform equivalent particle diameter, $D_{f D E M}=8.10^{-4} \mathrm{~m}$ and the porosity of the system $\varepsilon=0.665$. Note that subjected to a uniform distribution of particles and slippery boundary, the total fluid-particle interaction force was identical to all the particles in the 
system.

To evaluate the correlation between the inertial and viscous components of flow through a particulate media, the Reynolds number is useful according to Equation (10). Fig. $13 \mathrm{~b}$ represents the total fluid-particle interaction force $F_{f}$ acting on a fibre particle of the uniform porosity system in relation to different magnitudes of the superficial fluid velocity along the fibres. For a range of fluid velocities of less than $0.0013 \mathrm{~m} / \mathrm{s}$ corresponding to the Reynolds number lower than its unity (Darcy regime), the flow in this regime is not only laminar but also "creeping” flow (Trussell and Chang 1999), which have insignificant inertial contribution and a major component of the static buoyancy force. For a higher range of Reynolds number (Forcheimer regime), an increasing influence of inertial part on the total fluid-particle interaction force can be observed in the Fig. 13b. Besides, a gradual increase of the total fluid-particle interaction force occurs in a range of flow velocities of less than 0.01 $\mathrm{m} / \mathrm{s}$ before there is a sharp acceleration of such force due to a greater range of flow velocity. This increasing trend of the total fluid-particle interaction force also indicates a bigger role of the drag force in the total fluid force acting on the fibre particle when the fluid velocity progresses. Additionally, Fig. 13b shows the correlation between the total fluid-particle interaction force and fibre density which ranged from 1000 to $2500 \mathrm{~kg} / \mathrm{m}^{3}$. In this study the total fluid-particle interaction force was influenced slightly by the density of the fibre, but for different characteristics of fibres and fluid flow, the fluid interaction force acting on the fibre particles would be more heavily dependent on the density of fibres. Therefore the behaviour of fibres due to fluid flow must be considered in conjunction with their density and bond stiffness.

\section{Motion of fibres under fluid flow}

In order to observe the influence of fluid flow on the behaviour of fibre particles, one ends of fibres were fixed, while the other ends were free to move. A laminar (longitudinal) 
flow through a parallel fibre bundle with a range of density from 1000 to $2500 \mathrm{~kg} / \mathrm{m}^{3}$ and an equivalent diameter of the fibre particle, $D_{f D E M}=8.10^{-4} \mathrm{~m}$ was created in this section. In addition to the contact force which could be ignored in this investigation as explained in the previous section, the external forces acting on fibre particles included the fluid-particle interaction and gravitational forces (Fig. 13a). All fibres in the bundle were assigned the same set of characteristics and under a uniform fluid-particle interaction force that caused the fibres to act in an identical way. Motion of fibre particles with various values of the normal stiffness, $k_{b n}$ of the parallel bond and different magnitudes of fibre density was subjected to investigate in conjunction with different levels of flow velocity.

The axial strain of fibre that was considered as the ratio of the longitudinal displacement of the freely moving end to the total initial length of fibre is shown in the Fig. 14 in relation to the superficial velocity of flow. A higher velocity of laminar flow brings a bigger fluid interaction force exerting on the particles in the system, and that results in a more displacement of the fibre particles. The density of fibre shows a considerable impact on motion of the fibre particles in a range of flow velocities lower than $0.08 \mathrm{~m} / \mathrm{s}$, where the total fluid-particle interaction force is insignificant compared to the gravitational force. For a higher magnitude of flow velocity in which, the force generated by fluid flow acting on the fibre particles is dominant, the axial strain of fibre becomes less dependent on the density of fibre. Given a laminar flow, the computational results indicated that the longitudinal motion of fibre particles could be negligible for the fibres with a normal stiffness $k_{b n}$ of bond greater than $10^{6} \mathrm{~Pa} / \mathrm{m}$ (less than $1 \%$ of axial strain). Thereby, the motion of fibres must be evaluated in association with the stiffness of particles bonding.

\section{Model Limitations}

The study has a number of limitations. First, the parallel bond model might not accurately simulate the fibres which have predominantly ductile fracture. Improving the 
particle bonding would enable the coupling technique to be valid in a wider range of fibres. In addition, the modified contact model used to generate dense fibre packing cannot alter the fact that the DEM fibres were actually a series of spherical particles which were generally different from the real shape of fibres. Moreover, uniform fibre arrangement used in this study was a simple case to demonstrate the technique. Application of the method to real drainages which have predominantly disordered arrangement of fibres would be important. Finally, the hydraulic behaviour of fibrous media was investigated in the absence of soil particles and only fluid-fibre interaction was considered. While in many cases the hydraulic behaviour of fibrous geomaterials is inherent in the assembly of particles, rigorous experimental works such as filtration test are needed to further validate the coupling technique used in this study.

\section{Conclusions}

This paper describes and discusses a technique for numerically simulating the hydraulic behaviour of fibrous materials with respect to interaction between the fluid and solid phases. Fibre elements were simulated by the discrete element method while the fluid behaviour was captured by the finite volume method simultaneously. The following conclusions could be drawn:

(1) Fibrous geomaterials, particularly natural fibres such as jute and bamboo which have mainly linear stress-strain relationship and brittle fracture, can be simulated by the parallel bond model of a DEM framework. By adopting different sets of parameters for bonding, $k_{b n}$ and $k_{b s}$, various behaviours including soft to hard fibres could be modelled.

(2) A coupled CFD-DEM technique can be applied to describe the hydraulic behaviour of fluid flow through a fibrous medium with respect to the interaction between fluid-solid phases. For the case that fibres had insignificant motion, the longitudinal permeability 
obtained from CFD-DEM coupling technique was matched well compared to the conventional methods which considered fibres to be completely static during fluid flow. The hydraulic conductivity of fibrous materials modelled by the CFD-DEM technique, particularly for the medium and loose fibre bundle $(0.4<\varepsilon<1)$, agreed well with those obtained from other analytical and numerical studies in past literature, although there was a significant deviation in the hydraulic conductivity for the lower range of porosity $(\varepsilon<$ 0.4), where various distributions of fluid velocity were considered in recent studies. Moreover, with respect to the uniform distribution of velocity, the longitudinal permeability of the parallel fibre bundle was barely dependent on the arrangement ratio $S_{y} / S_{x}$

(3) The study also indicated that the coupling technique can describe the behaviour of fibre elements under fluid flow. The total fluid-particle interaction force was found to be heavily dependent on the flow velocity in a range of velocity greater than $0.01 \mathrm{~m} / \mathrm{s}$. Subjected to laminar longitudinal flow, the axial strain of fibres was found to be considerable when the normal stiffness of bond $k_{b n}$ was lower than $10^{6} \mathrm{~Pa} / \mathrm{m}$. The coupled CFD-DEM technique showed a reasonable dependence of the dynamic behaviour of fibre particles on fibre characteristics, including the density and the bond attributes while fluid flowing.

(4) The modified contact model applied in this study enabled the coupling technique to simulate the hydraulic conductivity of fibre systems with a low range of porosity (i.e., 0.2). This kind of treatment allowed the spherical particles in DEM to be placed closer to each other without generating an interaction force to achieve a fibre bundle with a higher density. 


\section{Acknowledgements}

The authors acknowledge the Australia Research Council for funding this research. The $1^{\text {st }}$ author's PhD scholarship is sponsored by the Australia Endeavour Scheme. The paper was edited by a Professional English Editor, Bill Clayton.

\section{References}

Bertrand, F., Leclaire, L. A. and Levecque, G. 2005. DEM-based models for the mixing of granular materials. Chemical Engineering Science, 60(8-9): 2517-2531. doi: http://dx.doi.org/10.1016/j.ces.2004.11.048.

Biswas, S., Ahsan, Q., Cenna, A., Hasan, M. and Hassan, A. 2013. Physical and mechanical properties of jute, bamboo and coir natural fiber. Fibers and Polymers, 14(10): 17261767. doi: 10.1007/s12221-013-1726-3.

Carman, P. C. 1937. Fluid flow through granular beds. Chemical Engineering Research and Design, 75, Supplement: S32-S48. doi: http://dx.doi.org/10.1016/S02638762(97)80003-2.

Chawla, K. K. 2007. Fibrous reinforcements for composites: overview. In Concise encyclopedia of composite materials. Edited by A. Mortensen. Elsevier Oxford, UK. pp. $140-146$.

Chen, F., Drumm, E. C. and Guiochon, G. 2011. Coupled discrete element and finite volume solution of two classical soil mechanics problems. Computers and Geotechnics, 38(5): 638-647. doi: http://dx.doi.org/10.1016/j.compgeo.2011.03.009.

Chen, X. and Papathanasiou, T. D. 2007. Micro-scale modeling of axial flow through unidirectional disordered fiber arrays. Composites Science and Technology, 67(7-8): 
1286-1293. doi: http://dx.doi.org/10.1016/j.compscitech.2006.10.011.

Cheng, Y. P., Nakata, Y. and Bolton, M. D. 2003. Discrete element simulation of crushable soil. Géotechnique, 53: 633-641. doi: 10.1680/geot.2003.53.7.633.

Cho, N., Martin, C. D. and Sego, D. C. 2007. A clumped particle model for rock. International Journal of Rock Mechanics and Mining Sciences, 44(7): 997-1010. doi: http://dx.doi.org/10.1016/j.ijrmms.2007.02.002.

Cundall, P. A. and Strack, O. D. L. 1979. A discrete numerical model for granular assemblies. Géotechnique, 29: 47-65.

Defoirdt, N., Biswas, S., Vriese, L. D., Tran, L. Q. N., Acker, J. V., Ahsan, Q., Gorbatikh, L., Vuure, A. V. and Verpoest, I. 2010. Assessment of the tensile properties of coir, bamboo and jute fibre. Composites Part A, 41: 588-595. doi: 10.1016/j.compositesa.2010.01.005.

Endruweit, A., Gommer, F. and Long, A. C. 2013. Stochastic analysis of fibre volume fraction and permeability in fibre bundles with random filament arrangement. Composites Part A: Applied Science and Manufacturing, 49: 109-118. doi: http://dx.doi.org/10.1016/j.compositesa.2013.02.012.

Gebart, B. R. 1992. Permeability of unidirectional reinforcements for RTM. Journal of Composite Materials, 26(8): 1100-1133. doi: 10.1177/002199839202600802.

Goniva, C., Kloss, C., Hager, A. and Pirker, S. 2010. An open source CFD-DEM perspectives. In Proceeding of the 5th OpenFOAM Workshop, Gothenburg, Sweden.

Gutowski, T. G., Morigaki, T. and Zhong Cai. 1987. The consolidation of laminate composites. Journal of Composite Materials, 21(2): 172-188. doi: $10.1177 / 002199838702100207$.

Happel, J. 1959. Viscous flow relative to arrays of cylinders. AIChE Journal, 5(2): 174-177. doi: 10.1002/aic.690050211. 
Jaiswal, J. N. and Singh, R. 2013. Comprehensive practical physics (Class XI). Laxmi Publications, New Delhi.

Kloss, C. and Goniva, C. 2010. LIGGGHTS: a new open source discrete element simulation software. In Proceeding of the 5th International Conference on Discrete Element Methods, London, UK.

Kloss, C., Goniva, C., Hager, A., Amberger, S. and Pirker, S. 2012. Models, algorithms and validation for opensource dem and cfdem. Progress in Computational Fluid Dynamics, an International Journal, 12(2): 140-152.

Li, Y., Xu, Y. and Thornton, C. 2005. A comparison of discrete element simulations and experiments for 'sandpiles' composed of spherical particles. Powder Technology, 160(3): 219-228. doi: http://dx.doi.org/10.1016/j.powtec.2005.09.002.

Lisjak, A. and Grasselli, G. 2014. A review of discrete modeling techniques for fracturing processes in discontinuous rock masses. Journal of Rock Mechanics and Geotechnical Engineering, 6(4): 301-314. doi: http://dx.doi.org/10.1016/j.jrmge.2013.12.007.

Mishra, S. P. 2000. A text book of fibre science and technology. New Age International Publishers, New Delhi.

Ngo, N. T., Indraratna, B. and Rujikiatkamjorn, C. 2014. DEM simulation of the behaviour of geogrid stabilised ballast fouled with coal. Computers and Geotechnics, 55: 224-231. doi: http://dx.doi.org/10.1016/j.compgeo.2013.09.008.

O'Sullivan, C. 2011. Particulate discrete element modelling: a geomechanics perspective. Spon Press (an imprint of Taylor \& Francis), London.

OpenFOAM. 2014. OpenFOAM user guide, Version 2.3.0.

Ozgumus, T., Mobedi, M. and Ozkol, U. 2014. Determination of kozeny constant based on porosity and pore to throat size ratio in porous medium with rectangular rods. Engineering Applications of Computational Fluid Mechanics, 8(2): 308-318. 
Potyondy, D. O. and Cundall, P. A. 2004. A bonded-particle model for rock. International Journal of Rock Mechanics and Mining Sciences, 41(8): 1329-1364. doi: http://dx.doi.org/10.1016/j.ijrmms.2004.09.011.

Shan, T. and Zhao, J. 2014. A coupled CFD-DEM analysis of granular flow impacting on a water reservoir. Acta Mechanica, 225(8): 2449-2470. doi: 10.1007/s00707-014-1119Z.

Smuts, E. M., Deglon, D. A. and Meyer, C. J. 2012. Methodology for CFD-DEM modelling of particulate suspension rheology. In Proceeding of the Ninth International Conference on CFD in the Minerals and Process Industries CSIRO, Melbourne, Australia.

Sullivan, R. R. 1942. Specific surface measurements on compact bundles of parallel fibers. Journal of Applied Physics, 13(11): 725-730. doi: doi:http://dx.doi.org/10.1063/1.1714824.

Tamayol, A. and Bahrami, M. 2008. Numerical investigation of flow in fibrous porous media. In ECI International Conference on Heat Transfer and Fluid Flow, Microscale, Whistler, Canada. pp. 21-26.

Tamayol, A. and Bahrami, M. 2009. Analytical determination of viscous permeability of fibrous porous media. International Journal of Heat and Mass Transfer, 52(9-10): 2407-2414. doi: http://dx.doi.org/10.1016/j.ijheatmasstransfer.2008.09.032.

Tamayol, A. and Bahrami, M. 2010. Parallel flow through ordered fibers: an analytical approach. Journal of Fluids Engineering, 132(11): 11450201-11450207. doi: $10.1115 / 1.4002169$.

Trussell, R. R. and Chang, M. 1999. Review of flow through porous media as applied to head loss in water filters. Journal of Environmental Engineering, 125(11): 998-1006. doi: doi:10.1061/(ASCE)0733-9372(1999)125:11(998). 
Tsuji, Y., Kawaguchi, T. and Tanaka, T. 1993. Discrete particle simulation of 2-dimensional fluidized-bed. Powder Technology, 77: 79-87.

Yazdchi, K., Srivastava, S. and Luding, S. 2011. Microstructural effects on the permeability of periodic fibrous porous media. International Journal of Multiphase Flow, 37(8): 956-966. doi: http://dx.doi.org/10.1016/j.ijmultiphaseflow.2011.05.003.

Zeghal, M. and El Shamy, U. 2004. A continuum-discrete hydromechanical analysis of granular deposit liquefaction. International Journal for Numerical and Analytical Methods in Geomechanics, 28(14): 1361-1383. doi: 10.1002/nag.390.

Zeghal, M. and El Shamy, U. 2008. Liquefaction of saturated loose and cemented granular soils. Powder 184(2): 254-265. Technology, doi: http://dx.doi.org/10.1016/j.powtec.2007.11.032.

Zhao, J. and Shan, T. 2013. Coupled CFD-DEM simulation of fluid-particle interaction in geomechanics. Powder Technology, 239: 248-258. doi: http://dx.doi.org/10.1016/j.powtec.2013.02.003.

Zhou, Z. Y., Kuang, S. B., Chu, K. W. and Yu, A. B. 2010. Discrete particle simulation of particle-fluid flow: model formulations and their applicability. Journal of Fluid Mechanics, 661: 482-510. doi: doi:10.1017/S002211201000306X.

Zhu, H. P., Zhou, Z. Y., Yang, R. Y. and Yu, A. B. 2007. Discrete particle simulation of particulate systems: theoretical developments. Chemical Engineering Science, 62(13): 3378-3396. doi: http://dx.doi.org/10.1016/j.ces.2006.12.089.

Zhu, H. P., Zhou, Z. Y., Yang, R. Y. and Yu, A. B. 2008. Discrete particle simulation of particulate systems: a review of major applications and findings. Chemical Engineering $\quad$ Science, $\quad$ 63(23): http://dx.doi.org/10.1016/j.ces.2008.08.006. 


\section{LIST OF FIGURES}

Fig. 1. Generalized stress-strain and fracture behaviours by DEM-Parallel Bond Model and by experiment on natural fibres (i.e., jute and bamboo)

Fig. 2. Scheme of parallel bond between two spherical particles

Fig. 3. Behaviour of a single fibre by DEM with different shear stiffness: (a) Hard fibre: $k_{b n}=$ $3 \times 10^{9} \mathrm{~Pa} / \mathrm{m} ; k_{b s}=1 \times 10^{9} \mathrm{~Pa} / \mathrm{m} ;(b)$ Soft fibre: $k_{b n}=3 \times 10^{9} \mathrm{~Pa} / \mathrm{m} ; k_{b s}=1 \times 10^{2} \mathrm{~Pa} / \mathrm{m}$

Fig. 4. Tension test for geogrid modelled by LIGGGHTS and PFC-3D

Fig. 5. Arrangement of fibres in: $(a)$ Rectangular and $(b)$ Triangular template

Fig. 6. Correlation of fibre structure in: (a) DEM and (b) Reality

Fig. 7. Concept of fibre particles contact: ( $a$ ) Original and (b) Modified contact models

Fig. 8. Fluid flow through fibrous porous media: $(a)$ Meshing fluid domain and $(b)$ Fibres in DEM coupled with CFD

Fig. 9. Average velocity, porosity and pressure drop through fibrous drain

Fig. 10. Velocity $\left(U_{f}\right)$ distribution of fluid flow through: (a) Squarely and $(b)$ Triangularly arranged fibres

Fig. 11. Dimensionless permeability of squarely arranged fibres over porosity by CFDEM with uniform distribution of fluid velocity and in comparison with other studies

Fig. 12. Dimensionless longitudinal permeability against arrangement ratio $S_{y} / S_{x}$

Fig. 13. Forces acting on a fibre particle under longitudinal fluid flow: (a) Potential forces acting on a fibre particle; $(b)$ Variation of forces subjected to increasing fluid velocity

Fig. 14. Axial strain of fibre under longitudinal flow in relation to superficial flow velocity 
Table 1 Parameters of the parallel bond for geogrid (Ngo et al. 2014)

\begin{tabular}{|l|l|}
\hline Radius of particle $R_{i}(\mathrm{~m})$ & $2 \times 10^{-3}$ \\
\hline Multiplier $\lambda$ & 0.5 \\
\hline Normal stiffness $k_{b n}(\mathrm{kPa} / \mathrm{m})$ & $5.68 \times 10^{8}$ \\
\hline Shear stiffness $k_{b s}(\mathrm{kPa} / \mathrm{m})$ & $5.68 \times 10^{8}$ \\
\hline Normal strength $\sigma_{b}(\mathrm{MPa})$ & 456 \\
\hline Shear strength $\tau_{b}(\mathrm{MPa})$ & 456 \\
\hline Density of particle $\rho_{p}\left(\mathrm{~kg} / \mathrm{m}^{3}\right)$ & 800 \\
\hline Radius of bond-disk $R_{b}(\mathrm{~m})$ & 0.001 \\
\hline
\end{tabular}




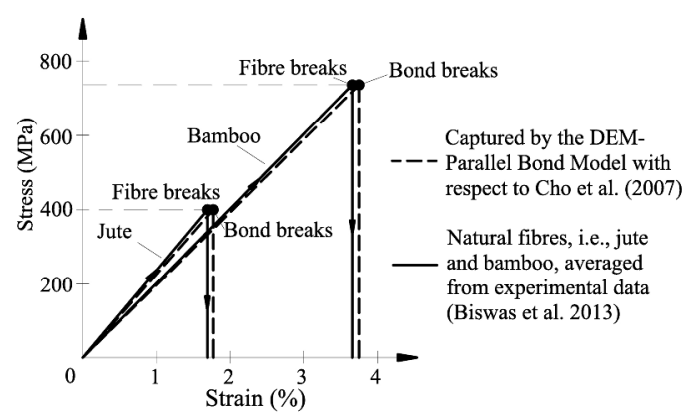

Fig. 1 Generalized stress-strain and fracture behaviours by DEM-Parallel Bond Model and by experiment on natural fibres (i.e., jute and bamboo)

Fig. 1 Generalized stress-strain and fracture behaviours by DEM-Parallel Bond Model and by experiment on natural fibres (i.e., jute and bamboo) $297 \times 420 \mathrm{~mm}(300 \times 300$ DPI $)$ 


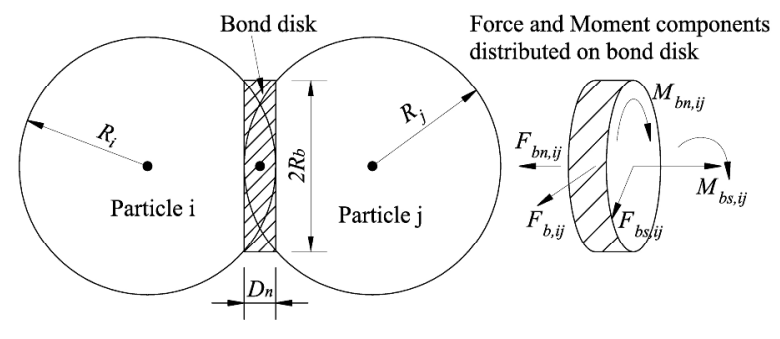

Fig. 2. Scheme of parallel bond between two spherical particles

Fig. 2. Scheme of parallel bond between two spherical particles $297 \times 420 \mathrm{~mm}$ (300 x 300 DPI) 


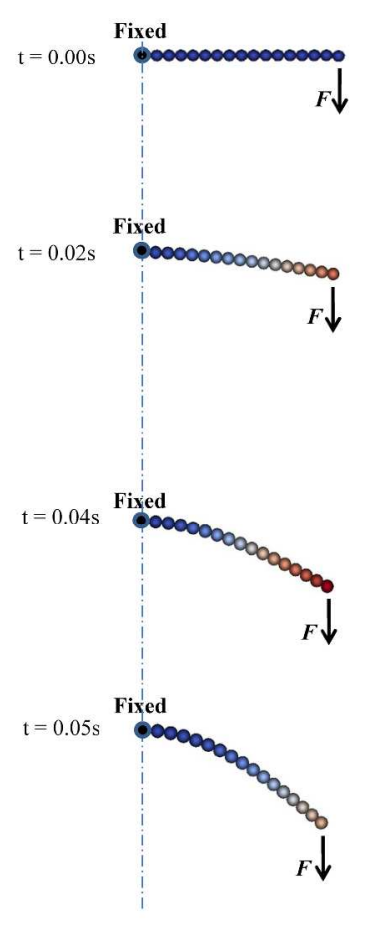

(a)

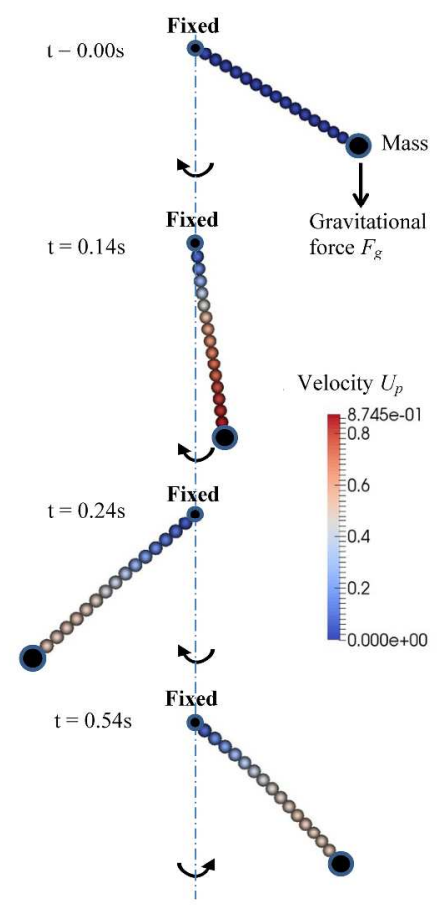

(b)

Fig. 3. Behaviour of a single fibre by DEM with different shear stiffness: (a) Hard fibre: $k_{b n}=$ $3 \times 10^{9} \mathrm{~Pa} / \mathrm{m} ; k_{b s}=1 \times 10^{9} \mathrm{~Pa} / \mathrm{m} ;(b)$ Soft fibre: $k_{b n}=3 \times 10^{9} \mathrm{~Pa} / \mathrm{m} ; k_{b s}=1 \times 10^{2} \mathrm{~Pa} / \mathrm{m}$

Fig. 3. Behaviour of a single fibre by DEM with different shear stiffness: (a) Hard fibre: $k b n=3 \times 109$ Pa/m; $\mathrm{kbs}=1 \times 109 \mathrm{~Pa} / \mathrm{m}$; (b) Soft fibre: $\mathrm{kbn}=3 \times 109 \mathrm{~Pa} / \mathrm{m}$; $\mathrm{kbs}=1 \times 102 \mathrm{~Pa} / \mathrm{m}$ 297x420mm (300 x 300 DPI) 


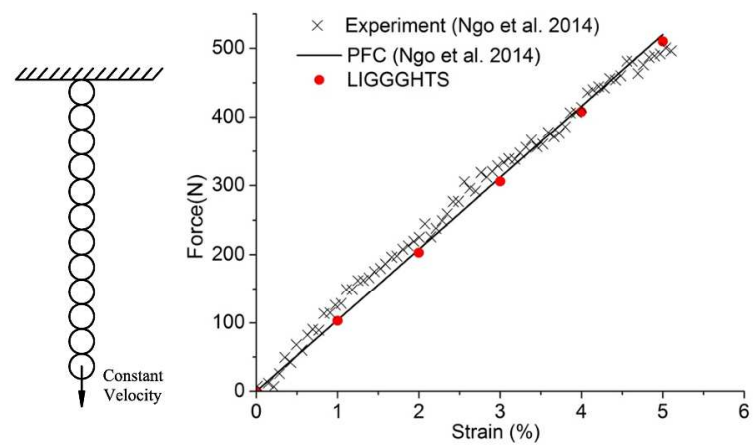

Fig. 4. Tension test for geogrid modelled by LIGGGHTS and PFC-3D

Fig. 4. Tension test for geogrid modelled by LIGGGHTS and PFC-3D $297 \times 420 \mathrm{~mm}$ (300 x 300 DPI) 


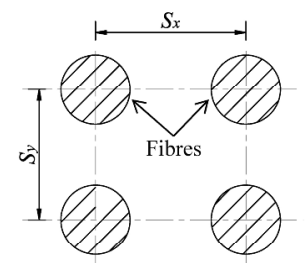

(a)

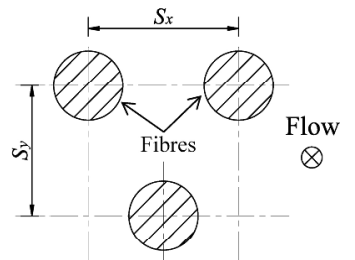

(b)

Fig. 5. Arrangement of fibres in: $(a)$ Rectangular and $(b)$ Triangular template

Fig. 5. Arrangement of fibres in: (a) Rectangular and (b) Triangular template $297 \times 420 \mathrm{~mm}(300 \times 300 \mathrm{DPI})$ 

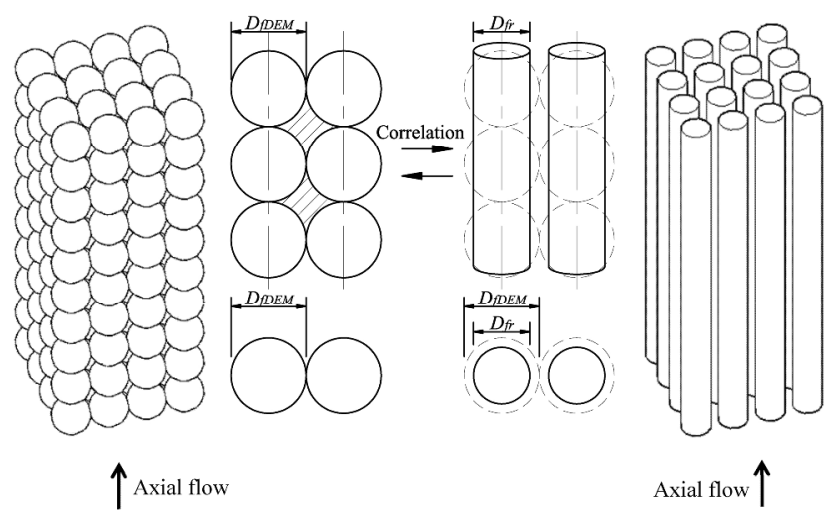

(a)

(b)

Fig. 6. Correlation of fibre structure in: (a) DEM and (b) Reality

Fig. 6. Correlation of fibre structure in: (a) DEM and (b) Reality $297 \times 420 \mathrm{~mm}(300 \times 300 \mathrm{DPI})$ 


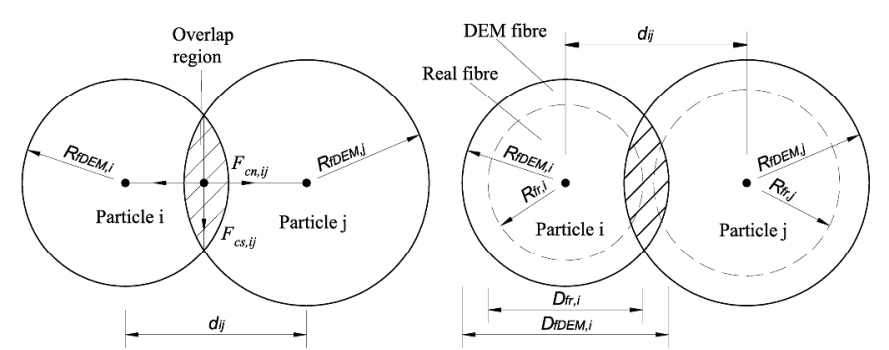

(a)

(b)

Fig. 7. Concept of fibre particles contact: (a) Original and (b) Modified contact models

Fig. 7. Concept of fibre particles contact: (a) Original and (b) Modified contact models $297 \times 420 \mathrm{~mm}(300 \times 300 \mathrm{DPI})$ 


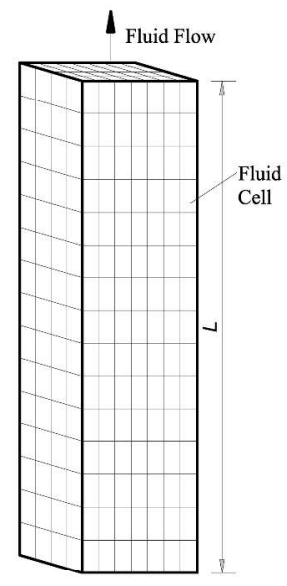

(a)

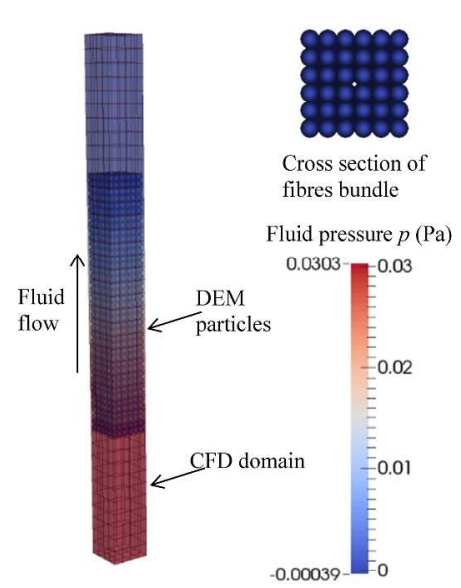

(b)

Fig. 8. Fluid flow through fibrous porous media: (a) Meshing fluid domain and (b) Fibres in DEM coupled with CFD

Fig. 8. Fluid flow through fibrous porous media: (a) Meshing fluid domain and (b) Fibres in DEM coupled with CFD

$297 \times 420 \mathrm{~mm}(300 \times 300$ DPI $)$ 


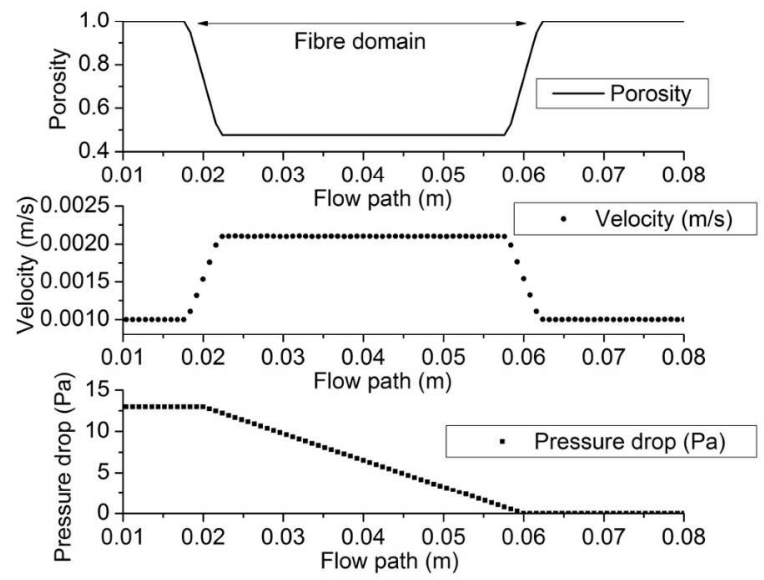

Fig. 9. Average velocity, porosity and pressure drop through fibrous drain

Fig. 9. Average velocity, porosity and pressure drop through fibrous drain 297x420mm (300 x 300 DPI) 


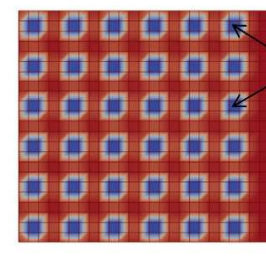

(a)

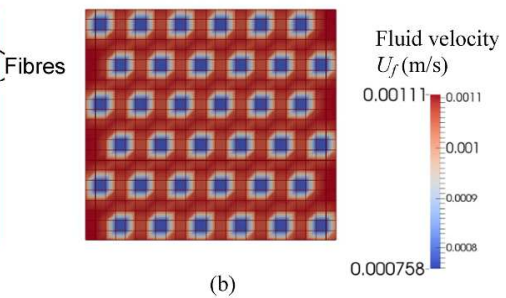

(b)

Fig. 10. Velocity $\left(U_{f}\right)$ distribution of fluid flow through: (a) Squarely and $(b)$ Triangularly

arranged fibres

Fig. 10. Velocity (Uf ) distribution of fluid flow through: (a) Squarely and (b) Triangularly arranged fibres $297 \times 420 \mathrm{~mm}(300 \times 300 \mathrm{DPI})$ 


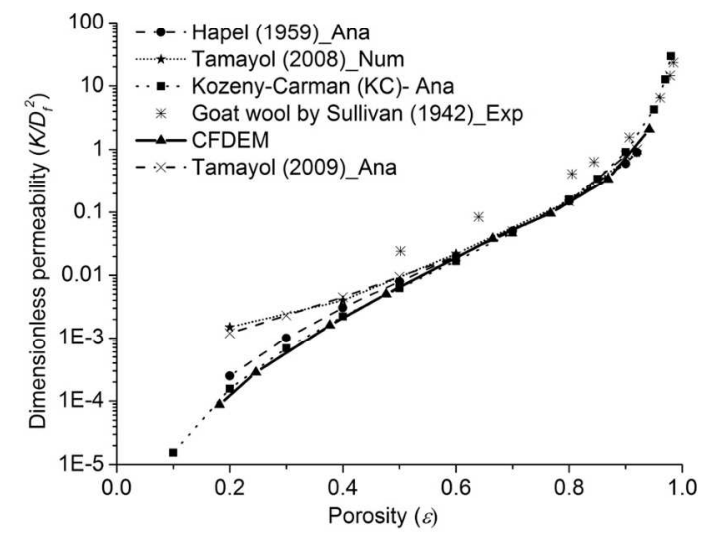

Fig. 11. Dimensionless permeability of squarely arranged fibres over porosity by CFDEM

with uniform distribution of fluid velocity and in comparison with other studies

Fig. 11. Dimensionless permeability of squarely arranged fibres over porosity by CFDEM with uniform distribution of fluid velocity and in comparison with other studies 297x420mm (300 x 300 DPI) 


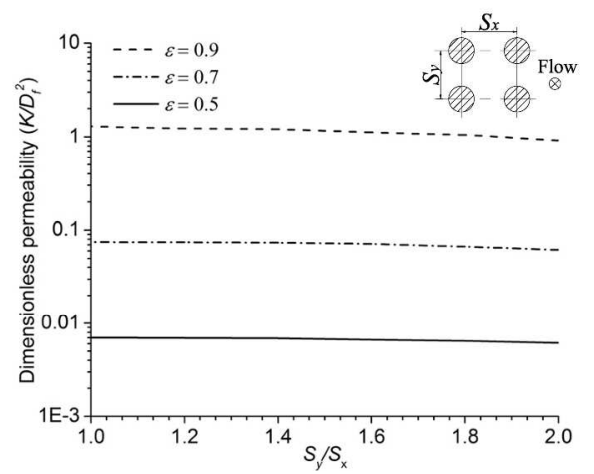

Fig. 12. Dimensionless longitudinal permeability against arrangement ratio $S_{y} / S_{x}$

Fig. 12. Dimensionless longitudinal permeability against arrangement ratio Sy/Sx $297 \times 420 \mathrm{~mm}(300 \times 300 \mathrm{DPI})$ 


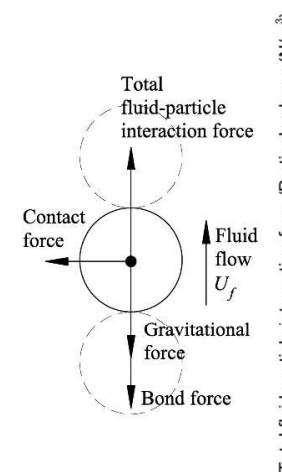

(a)

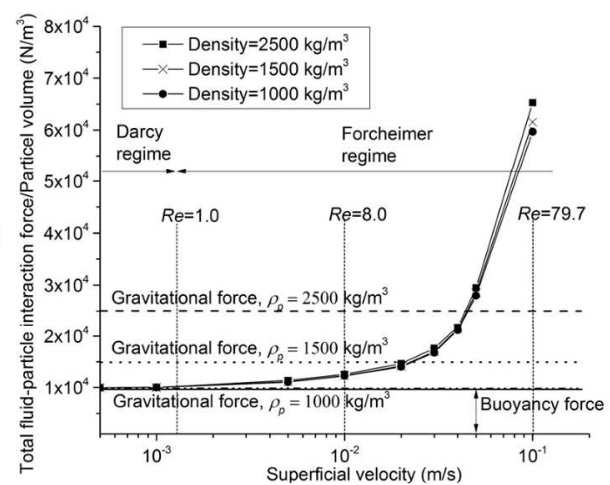

(b)

Fig. 13. Forces acting on a fibre particle under longitudinal fluid flow: (a) Potential forces acting on a fibre particle; (b) Variation of forces subjected to increasing fluid velocity

Fig. 13. Forces acting on a fibre particle under longitudinal fluid flow: (a) Potential forces acting on a fibre particle; (b) Variation of forces subjected to increasing fluid velocity $297 \times 420 \mathrm{~mm}(300 \times 300$ DPI) 


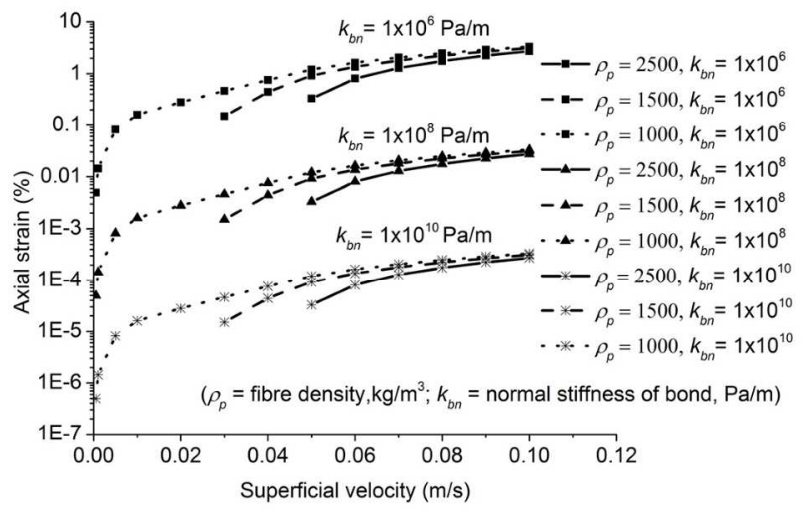

Fig. 14. Axial strain of fibre under longitudinal flow in relation to superficial flow velocity

Fig. 14. Axial strain of fibre under longitudinal flow in relation to superficial flow velocity $297 \times 420 \mathrm{~mm}(300 \times 300$ DPI) 